\title{
El efecto de las actividades de tiempo libre y del engagement escolar sobre el rendimiento académico de las personas jóvenes
}

\section{The effect of leisure activities and school engagement on school attainment of youth}

\author{
Arnau Palou ${ }^{1}$, Ona Valls ${ }^{2}$, Rafael Merino ${ }^{3}$ \\ ${ }^{1}$ Universidad Autónoma de Barcelona. arnau.palou@uab.cat \\ ${ }^{2}$ Universidad Autónoma de Barcelona. ona.valls@uab.cat \\ ${ }^{3}$ Universidad Autónoma de Barcelona.rafael.merino@uab.cat
}

Recibido: 16/10/2019

Aceptado: 10/04/2020

Copyright (C)

Facultad de CC. de la Educación y Deporte. Universidad de Vigo

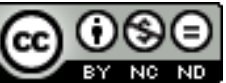

Dirección de contacto:

Arnau Palou

Facultad de Ciencias Políticas y de Sociología Edificio B. Campus de la UAB (Despacho B3073)

08193 Bellaterra (Cerdanyola del Vallès)

Barcelona

\begin{abstract}
Resumen
El presente artículo trata de averiguar las relaciones que se establecen entre el origen social, las actividades de tiempo libre, el rendimiento académico y el engagement escolar del alumnado. El principal objetivo de esta investigación es analizar si las actividades de tiempo libre y el engagement escolar pueden compensar el efecto del origen social sobre el rendimiento académico. La investigación utiliza una muestra de 2.056 jóvenes de 27 centros diferentes de la ciudad de Barcelona y analiza los datos a través de un modelo de ecuaciones estructurales. Los resultados muestran que las actividades de relación y de pantalla están asociadas a un incremento del logro escolar, y las actividades deportivas y culturales a un aumento del engagement emocional y cognitivo. Las actividades culturales también se asocian a un mejor engagement conductual, un tipo de engagement que tiene un efecto positivo sobre el logro escolar y que puede mediar el efecto de sexo y de origen inmigrante sobre el rendimiento académico.
\end{abstract}

\section{Palabras clave}

Actividades de Tiempo Libre, Engagement Escolar, Rendimiento Académico, Desigualdades Educativas, Modelos de Ecuaciones Estructurales

\begin{abstract}
This paper aims to analyze the relations between social background, leisure activities, school attainment and school engagement. The main objective of this research is to examine whether leisure activities and school engagement can compensate for the effect of social background on school attainment. This research uses a sample of 2.056 10th grade students from 27 different centers within the city of Barcelona. Data processing is done by structural equation modeling. The results show that relational and digital activities are associated with an improvement in school attainment while sport and cultural activities are associated with an increase in emotional and cognitive engagement. Cultural activities are also related to a better
\end{abstract}


behavioral engagement, a type of engagement that has a positive effect on school attainment and can compensate for the effect of gender and immigrant background on school attainment.

\section{Key Words}

Leisure Activities, School Attainment, School Engagement, Educational Inequalities, Structural Equation Modeling

\section{INTRODUCCIÓN}

El papel del ocio en la sociedad ha sido un tema investigado desde la antigüedad y con especial énfasis a partir del siglo XIX. Thorstein Veblen, en su emblemática obra "Teoría de la clase ociosa", analizó el ocio como un mecanismo de "emulación pecuniaria” (Veblen, 2004), destacando su función de distinción social entre la burguesía de principios del siglo XX. Años después, Dumazedier, uno de los padres de la Sociología del Ocio, centró su obra en descubrir las causas y las consecuencias de la aparición de la "civilización del ocio", en la Francia de los años cincuenta del siglo pasado, momento en el cual el ocio adquirió un fuerte valor social, tanto para la burguesía como para la clase obrera de la época (Dumazedier, 1968).

El ocio, entendido como el contenido que los individuos dan a su tiempo libre (Merino, 2007), generó una nueva preocupación en relación a cómo la sociedad iba a utilizarlo en este contexto de democratización y de expansión de sus opciones. Dumazedier consideraba esta preocupación un "problema social" que precisaba ser analizado en profundidad. Con este objetivo clasificó el ocio entre el ocio activo y el ocio pasivo. En el primero colocó aquellas actividades que generaban nuevos procesos de aprendizaje informal y voluntario y que implicaban una actitud consciente, participativa y selectiva por parte de los individuos. El ocio pasivo lo relacionó con las actividades no reflexivas, aquellas que no precisaban ningún esfuerzo por parte de los individuos, ni les aportaban nuevos conocimientos ni habilidades (Dumazedier, 1968). Desde entonces, numerosos autores (Merino, 2007; Putnam, 2015; Nordlander, 2016) han abordado el tema del ocio desde distintos enfoques, a partir de diferentes clasificaciones y en busca de varios objetivos.

Dentro de la sociología de la juventud, se ha popularizado el estudio de las habilidades no cognitivas -como las habilidades sociales y emocionales- adquiridas y desarrolladas durante el tiempo libre de las personas jóvenes, con el objetivo de intentar operativizar su transferibilidad en el ámbito formativo y laboral. Estos estudios parten de la idea de que ciertas actividades realizadas durante el tiempo libre pueden convertirse en una fuente de competencias útiles para las trayectorias formativas y laborales de los y las jóvenes (Moreno, 2009). Al mismo tiempo, asocian estas actividades con espacios de participación social que contribuyen a su desarrollo integral y a la interacción entre iguales.

En este artículo, se utilizará el concepto de actividades de tiempo libre (ATL) en vez del de actividades de ocio, ya que este es un concepto demasiado amplio y confuso. Las actividades de tiempo libre se definen aquí como aquellas actividades que practican los individuos, condicionados por su posición social y por las oportunidades de su entorno, 
en su tiempo exento de obligaciones productivas y familiares. Concretamente, se investigarán las actividades de tiempo libre de las personas jóvenes.

Con el fin de obtener más información sobre el potencial de las actividades de tiempo libre es importante seguir investigando este campo de la Sociología, situado al margen de la escuela y del trabajo, pero con un gran impacto en ambas áreas de la vida de las personas jóvenes. En este sentido, la investigación busca contribuir a tal empresa desde una perspectiva innovadora, añadiendo la variable de engagement como mediadora del peso explicativo que pueden tener las actividades de tiempo libre sobre el logro escolar.

La estructura de este artículo es la siguiente. En primer lugar, se repasan las aportaciones teóricas y empíricas más relevantes sobre las actividades de tiempo libre y sobre el engagement escolar. A continuación, se explica el modelo de análisis que se va a seguir y las variables utilizadas en este modelo. En el tercer apartado se presenta la explotación de los datos y en el cuarto se comentan los resultados obtenidos, los esperados y los sorpresivos. Finalmente, aparecen las conclusiones, donde se contrastan las hipótesis, se mencionan las limitaciones metodológicas de este trabajo y se recomiendan posibles puntos de continuidad para futuras investigaciones. En el cierre, se comentan las posibles implicaciones sociopolíticas derivadas de los resultados de esta investigación.

\subsection{Las actividades de tiempo libre de las personas jóvenes}

En España, las actividades de tiempo libre representan un elemento central en la vida de las personas jóvenes (Benedicto y otros, 2017). Las actividades desarrolladas fuera de la escuela y del trabajo aportan significados y generan espacios donde pueden crecer y desarrollarse como personas (Eccles y Gootman, 2002). Estas actividades tienen un papel destacado en la construcción de sus estilos de vida; un hecho relevante si tenemos en cuenta que el estilo de vida tiene un fuerte impacto sobre las trayectorias formativas de los y las jóvenes (Archer, 2007).

Varios estudios han demostrado que hay ciertas actividades de tiempo libre asociadas a un mejor rendimiento escolar (Putnam, 2015) y a una vinculación positiva con los estudios, con los centros educativos y con el profesorado (Zaff, Moore, Papillo y Williams, 2003). La implicación en actividades extraescolares, que son actividades de tiempo libre desarrolladas dentro del centro educativo y supervisadas por adultos, se ha relacionado con tasas bajas de abandono escolar y con una reducción de conductas de riesgo, de acciones delictivas y de abuso de drogas. Las actividades extraescolares también amplían la red social de las personas jóvenes y pueden ofrecer mentores para aquellas con menos soporte familiar (Putnam, 2015). Otras investigaciones destacan la relación positiva entre estas actividades y algunos beneficios psicológicos como la autoestima o la resiliencia (Eccles, Barber, Stone y Hunt, 2003). La literatura también señala algunos efectos perniciosos de estas actividades sobre los itinerarios formativos y laborales de las personas jóvenes. El tipo de actividad y la frecuencia de la práctica explican gran parte de esta dimensión perjudicial. Por ejemplo, existe una relación entre participar en competiciones deportivas altamente competitivas y el aumento del estrés y de la ansiedad del alumnado (Fredricks y Eccles, 2006).

La literatura sociológica ha demostrado que existe una división social dentro de las actividades de tiempo libre (Shildrick y MacDonald, 2006). Dependiendo del sexo, del nivel formativo de los progenitores, de sus categorías laborales, y de su lugar de 
residencia, el joven o la joven tenderán a practicar un cierto tipo de actividades de tiempo libre. Estas variables, entre otros factores, crean unas barreras de acceso muy presentes en el caso de las personas jóvenes. Se trata de un conjunto de barreras económicas, como el pay to play (Putnam, 2015), sociales y educativas, que impiden el acceso de algunas personas a ciertas actividades. Paradójicamente, tal como defienden algunos autores (Nordlander, 2016; Putnam, 2015), el alumnado de familias con menos recursos económicos y culturales puede beneficiarse más de este tipo de actividades que el alumnado procedente de familias más acomodadas.

Un ejemplo de esta división social lo encontramos en algunos estudios sobre los beneficios del deporte, que han demostrado cómo las prácticas deportivas varían según la clase social de las personas jóvenes, tanto en sus prácticas como en sus efectos. Hay deportes restringidos a una determinada posición social, no solo por las barreras económicas, sino también por los valores implícitos en cada deporte y por los intereses de cada joven. Los y las jóvenes con un nivel socioeconómico bajo tienen más probabilidades de abandonar antes la práctica deportiva que sus coetáneos y coetáneas con niveles socioeconómicos superiores. Este fenómeno es debido a que tienen una percepción de los beneficios del deporte inferior a la que tiene el alumnado de clase media y alta (Fraguela, De-Juanas y Franco, 2017).

No obstante, hay actividades de tiempo libre de carácter generacional, no tan condicionadas por la clase social del alumnado, como por ejemplo navegar por internet o salir con los amigos. Estas actividades las realizan una gran mayoría de las personas jóvenes en España (Benedicto y otros, 2017). Los y las jóvenes navegan por internet desde varias plataformas -teléfono, tableta, ordenador, etc.- y lo hacen interconectados en red. De ahí el nombre de e-ocio, un tipo de ocio "móvil, transmedia, multisoporte y casual, cuya finalidad no es otra que informarse, comunicarse y entretenerse” (Viñals, 2016:11). Tanto el e-ocio como salir con los amigos forman parte de un tipo de actividades sociales que implican estar en compañía, ya sea presencial o virtual.

\subsection{El engagement escolar: tipos y efectos}

El engagement es un concepto ampliamente utilizado pero su definición varía en la literatura (Fredricks, Blumenfeld y Paris, 2004). Esta investigación utiliza este anglicismo por falta de consenso dentro de la academia a la hora de traducir el concepto. En España, algunos autores hablan de implicación (Ramos-Díaz, RodríguezFernández, Ros y Antonio-Aguirre, 2016) y otros de vinculación (Tarabini, Curran, Montes y Parcerisa, 2015) o de sentimiento de pertinencia. Con el fin de evitar confusiones y englobar las diferentes acepciones en una misma palabra, se opta por utilizar el concepto inglés de engagement.

La literatura disponible sobre el tema coincide en destacar la interrelación entre el engagement escolar, el desarrollo juvenil y el logro académico, y muestra cómo una alta implicación con la escuela puede mejorar el rendimiento académico (Juvonen, Espinoza y Knifsend, 2012) y viceversa: un fracaso escolar puede incrementar una conducta de riesgo con la edad (Finn, 1989). Los expertos también coinciden en señalar las actividades extracurriculares como un elemento clave para contribuir a aumentar el sentimiento de identificación con la escuela y las consideran un mecanismo eficaz para mejorar el engagement del alumnado con menos desempeño académico (Finn, 1989). Estas actividades también se asocian a trayectorias formativas con menos probabilidad 
de abandono (Jimerson, Campos y Greif, 2003). Algunos autores subrayan el papel del grupo de amigos tanto para implicarse con las actividades extracurriculares como con la escuela en general. Así, una relación positiva con el grupo de pares facilita el sentimiento de pertenencia con la escuela y evita experiencias negativas como el rechazo o el bullying, dos factores relacionados con la desvinculación académica (Juvonen et al., 2012).

Para Finn, uno de los autores más citados en esta materia, el engagement escolar se articula a través de la participación académica y de la identificación con la escuela (Finn, 1989). Pero en la conceptualización del engagement se tienen en cuenta distintos aspectos; dependiendo de la investigación, podemos encontrar el rendimiento académico, el comportamiento en clase o la implicación en actividades extracurriculares, entre otros (Jimerson et al., 2003). A pesar de esto, existe consenso en entender el engagement como un concepto multidimensional (Fredricks et al., 2004) y, mayoritariamente, tanto en sociología de la educación como en psicología, se diferencian tres dimensiones de engagement: el conductual, el emocional y el cognitivo (Janosz, Archambault, Morizot y Pagani, 2008).

El engagement conductual está relacionado con conductas específicas de los estudiantes, tales como la asistencia, los problemas de comportamiento y la participación en la escuela o en actividades escolares. Los jóvenes tienen un alto nivel de engagement conductual cuando asisten regularmente a clase, no se meten en problemas y van a clase con una buena predisposición para aprender (Finn y Voelkl, 1993). También hay investigadores que miden el engagement de conducta de los estudiantes basándose en la mala conducta (Janosz et al., 2008). El engagement emocional hace referencia a la identificación expresiva e instrumental con la escuela; una identificación que varía según los sentimientos que tienen los y las estudiantes respecto a la escuela, al grupo de pares y a los profesores/as. Centra el interés en la escuela y denota el alcance de las reacciones positivas y negativas hacia los maestros, los compañeros de clase y la escuela, incluyendo el sentido de pertenencia e identificación con la escuela y con las distintas materias (Boekaerts, 2016). En la literatura, el engagement cognitivo se ha medido de distintas formas. Por un lado, aspectos observables como el tiempo en la tarea, la participación en clase, la finalización de la tarea (Appleton et al., 2006), se extraen a menudo de las observaciones de los profesores sobre el comportamiento de los estudiantes en el aula (Finn y Zimmer, 2012). Por otro lado, se han utilizado los autoinformes de los estudiantes que se centran en el trabajo escolar y la persistencia cuando el contenido es difícil (Finn y Rock, 1997; Reeve y Tseng, 2011). Algunos investigadores también hicieron hincapié en la autorregulación del esfuerzo académico por parte de los estudiantes (Fredricks et al., 2004). Las tres dimensiones del engagement están relacionadas entre sí y representan un factor explicativo de la experiencia escolar de las personas jóvenes.

\section{ESTRATEGIA ANALÍTICA}

El modelo de análisis está diseñado con el fin de ver las relaciones directas e indirectas que se establecen entre las actividades de tiempo libre, el engagement escolar, el rendimiento académico -medido a través de una Prueba de Competencias Básicas 
(PCB) - y las variables sociodemográficas de sexo, origen inmigrante y nivel formativo familiar. En primer lugar, se analiza el efecto directo de las variables sociodemográficas sobre el engagement, sobre las actividades de tiempo libre y sobre la PCB. En segundo lugar, se observa el efecto de las actividades de tiempo libre sobre la PCB y el efecto del engagement sobre la PCB. Dentro de las relaciones indirectas o mediadas, se analiza el efecto del engagement sobre la PCB, mediado por las variables sociodemográficas y por las actividades de tiempo libre. Las otras relaciones indirectas analizadas son el efecto de las actividades de tiempo libre sobre la PCB, mediado por las variables sociodemográficas y por el engagement, así como el efecto de las variables sociodemográficas sobre la PCB, mediadas por el engagement y por las actividades de tiempo libre. También se analiza el efecto de las variables sociodemográficas sobre el engagement escolar, mediado por las actividades de tiempo libre.

\subsection{Modelo de análisis}

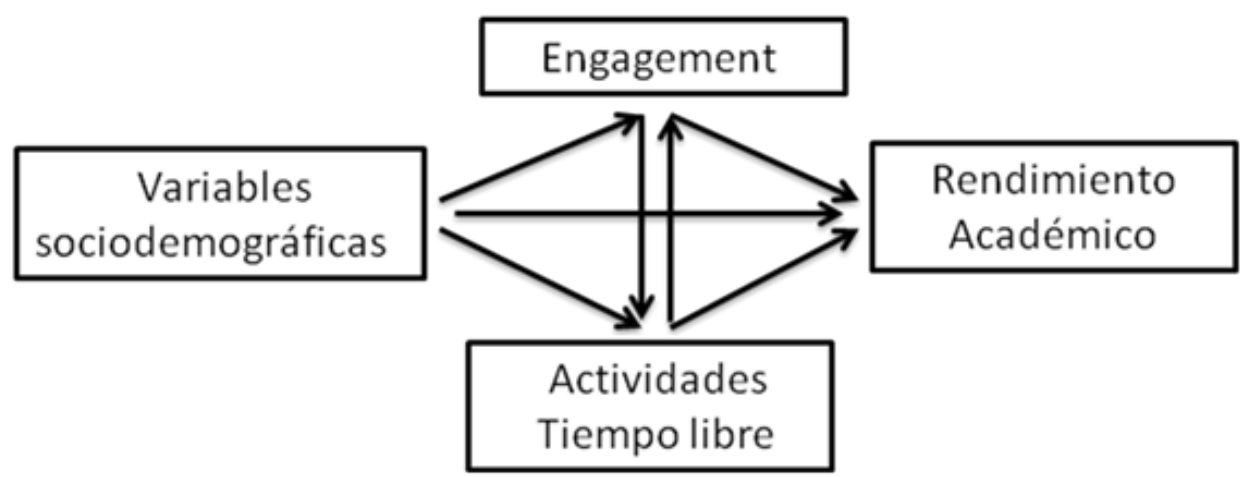

Figura 1. Modelo de Análisis

A partir de este modelo se plantean las siguientes hipótesis:

1. Según el tipo de actividad de tiempo libre del alumnado, esta actividad tendrá un efecto positivo o negativo en su rendimiento académico.

2. Según el tipo de actividad de tiempo libre del alumnado, esta actividad tendrá un efecto positivo o negativo en su engagement escolar.

3. El engagement puede compensar el efecto de las variables sociodemográficas sobre el rendimiento académico.

4. Las actividades de tiempo libre pueden incrementar el engagement $y$, de forma indirecta, aumentar el rendimiento académico del alumnado.

5. La incidencia de las actividades de tiempo libre sobre el rendimiento académico es mayor cuando la relación entre el origen social y el rendimiento es más negativa.

Para corroborar o refutar estas hipótesis de investigación se realizará un modelo de ecuaciones estructurales, una técnica que en este tipo de operaciones supera el análisis de regresión y el análisis factorial (López-Roldán y Fachelli, 2015). En este modelo aparecen variables manifiestas y variables latentes, que son las que se crean a través del análisis factorial. Las primeras son las que están relacionadas con las notas (PCB) y con las variables sociodemográficas, como son el origen inmigrante, el nivel formativo familiar y el sexo. Como variables latentes se crean tres subdimensiones de engagement -conductual, cognitivo y emocional- y tres tipos de actividades de tiempo libre deportivas, culturales, relacionales y de pantalla-. 


\subsection{Muestra}

Los datos utilizados en esta investigación proceden del International Study of City Youth (ISCY Project), un proyecto longitudinal que analiza y compara los itinerarios de las personas jóvenes de distintas ciudades del mundo una vez han terminado la educación secundaria obligatoria. Este trabajo utiliza los datos recopilados durante la primera ola del estudio realizado en la ciudad de Barcelona. Se trata de una muestra representativa de la ciudad de Barcelona compuesta por 2.056 estudiantes de 27 centros diferentes (Tabla 4 del Anexo) que durante el curso 2013-2014 cursaban $4^{\circ}$ de la ESO. Se hizo una selección de los centros teniendo en cuenta su titularidad (públicos y privados concertados) y su complejidad social, con el fin de que la muestra fuera representativa de los centros académicos de la ciudad de Barcelona. Para medir la complejidad de los centros se utiliza el indicador del Departamento de Educación que clasifica los centros en función de su contexto socioeconómico y del tipo de alumnado. Por ejemplo, tiene en cuenta el número de alumnos con becas comedor o el número de alumnos con necesidades educativas específicas.

\subsubsection{Variable dependiente}

Pruebas de competencias básicas (PCB). Esta variable representa una medida objetiva del logro escolar. Se trata de un test externo a la escuela para medir las competencias, las habilidades y los aprendizajes del alumnado. El test sustituye las notas de los exámenes habituales porque la encuesta se hizo antes de los exámenes finales. Se construyó un índice sintético a partir de los resultados en Lengua y Matemáticas.

\subsubsection{Variable independiente}

Actividades de tiempo libre (ATL). Las actividades de tiempo libre de la encuesta fueron seleccionadas por su relevancia en estudios anteriores (citados en el apartado teórico) a la hora de explicar el rendimiento académico y el engagement escolar de las personas jóvenes. Las preguntas de la encuesta hacían referencia a las siguientes actividades:

- Leer un libro por placer

- Practicar un deporte

- Hacer ejercicio para mantenerse en forma

- Mirar la TV

- Jugar a videojuegos

- Navegar por internet

- Tocar un instrumento musical

- Pasar el rato con los amigos

Las posibles respuestas eran: "Nunca”, "Menos de una vez a la semana”, "Una vez a la semana”, “Casi cada día”, “Cada día 2 o menos horas”, “Cada día dos horas o más”. Para reducir el número de variables y encontrar los factores definitorios de las actividades de tiempo libre se realizó un Análisis Factorial exploratorio con el software Mplus. Una vez eliminado el ítem de "Jugar a Videojuegos" porque no encajaba en el 
factorial, la bondad del modelo era buena, teniendo en cuenta distintos indicadores como el Root Mean Square Error Of Approximation -RMSEA- $(0,027)$, el CFI $(0,999)$ y el TLI (0,997). El resultado (adjunto en el anexo) muestra tres tipos de actividades: Actividades deportivas, Actividades culturales y Actividades de relación y de pantalla.

La justificación teórica de la agrupación en un mismo factor de "Mirar la TV", "Navegar por internet” y "Pasar el rato con los amigos" la encontramos en la literatura citada anteriormente. Tanto el e-ocio como pasar el rato con los amigos de forma presencial tienen un componente de relación muy fuerte. De hecho, según el último Informe Juventud (Benedicto y otros, 2017), uno de los motivos principales por el que las personas jóvenes se conectan a internet es para comunicarse con otras personas.

La variable de engagement se construyó a través de un análisis factorial con el programa Mplus. Las pruebas del ajuste del modelo dieron un valor estimado del RMSEA de 0,041, un valor de CFI del 0,920 y de TLI de 0,904. De esta manera la validez del modelo queda confirmada, dado que la bondad del modelo es adecuada si el coeficiente CFI y TLI es mayor de ,90 (Byrne, 2001) y si el coeficiente RMSEA es menor de ,08 (Kline, 1998). Una vez confirmada la bondad del modelo se aceptaron los siguientes tipos de engagement:

Engagement de conducta. Esta variable se generó basándose en 5 preguntas de tipo Likert relacionadas con la asistencia a clase y con la disciplina. El rango de respuesta era de 4: de "Nunca” a “5 veces o más”.

Asistencia

1. Me he saltado una clase sin permiso

2. He faltado un día sin permiso

Disciplina

3. Frecuentemente me meto en problemas

4. He sido castigado

5. He tenido problemas con algún profesor por mi conducta

Engagement emocional. Esta variable se generó basándose en 8 preguntas de tipo Likert relacionadas con la identificación expresiva e instrumental con la escuela. El rango de respuesta era de 4: de "Muy en desacuerdo" a "Muy de acuerdo".

Identificación instrumental

1. Trabajar duro en la escuela ayuda al éxito laboral

2. Lo que aprendemos en clase es necesario para tener éxito en el futuro

3. La escuela me prepara para tener un proyecto de vida

4. La escuela me enseña coses valiosas

\section{Identificación expresiva}

5. Me gusta estar en la escuela

6. Tengo buena relación con la mayoría de los profesores/as

7. Cuando termine la escuela tendré muy buenos recuerdos

8. Me siento seguro en la escuela 
Engagement cognitivo. Esta variable se generó basándose en 6 preguntas de tipo Likert relacionadas con el esfuerzo y la perseverancia en la escuela. El rango de respuesta era de 4: de "Muy en desacuerdo" a "Muy de acuerdo".

Esfuerzo

1. Siempre intento hacer las cosas lo mejor posible

2. En clase me esfuerzo al máximo

3. En clase doy lo mejor de mí

\section{Perseverancia}

5. Soy un/a estudiante muy trabajador/a

6. Siempre entrego los deberes a tiempo

7. Soy constante hasta terminar el trabajo

\subsubsection{Variables de control}

Sexo. Variable dicotómica: hombre o mujer.

Nivel Formativo Familiar. Variable construida a partir del nivel formativo más alto de los progenitores y dividida en tres niveles: sin estudios o con estudios obligatorios, con estudios postobligatorios y con estudios universitarios.

Origen inmigrante. Variable dicotómica: autóctono o inmigrante. En la categoría de inmigrante se encuentran tanto el alumnado que ha nacido en España, pero sus padres nacieron en el extranjero, como el alumnado que ha nacido en el extranjero.

\subsection{Análisis}

Los resultados del análisis emergen una vez se ha ejecutado el modelo de ecuaciones estructurales. Las pruebas estadísticas guardadas en el anexo muestran cómo el valor estimado del RMSEA (0.041) y del Standardized Root Mean Square Residual SRMSR-(0,041) son menores que 0,08 , y tanto el CFI $(0,920)$ como el TLI $(0,904)$ son mayores de 0,90 .

\section{RESULTADOS}

La Tabla 1 muestra las relaciones directas establecidas por el modelo entre las actividades de tiempo libre, las tres dimensiones de engagement, las variables sociodemográficas y el rendimiento académico (PCB). Al final de la tabla aparecen las relaciones establecidas entre las variables sociodemográficas y el rendimiento académico. Por un lado, se observa cómo las chicas tienen un rendimiento inferior al de los chicos. Se trata de una diferencia de medias muy pequeña que solo es significativa a través de la Prueba de Competencias Básicas (PCB), ya que, si se mide el rendimiento a través de la variable "notas del último curso", son las chicas quienes presentan una media de notas superior. Por otro lado, aparece que el alumnado autóctono tiene una media de notas superior a la del alumnado inmigrante, se calcule a través del PCB o de las “notas del último curso”. En este caso la desviación en el coeficiente es de 0,310. En relación al nivel formativo familiar, los hijos e hijas de padres con estudios obligatorios 
y post obligatorios tienen un rendimiento inferior al de los hijos e hijas de progenitores con estudios universitarios.

\section{EFECTOS DIRECTOS}

\begin{tabular}{|c|c|c|c|c|c|}
\hline \multicolumn{6}{|c|}{ ATL sobre engagement } \\
\hline Conductual & Estim. & Emocional & Estim. & Cognitivo & Estim. \\
\hline DEPORTIVA & $-0,038$ & DEPORTIVA & $0,090 * *$ & DEPORTIVA & $0,097 * *$ \\
\hline REL_PANT & $-0,346 * * *$ & REL_PANT & $-0,009$ & REL_PANT & $-0,141 * * *$ \\
\hline CULTURAL & $0,134 * * *$ & CULTURAL & $0,092 * *$ & CULTURAL & $0,135^{* * *}$ \\
\hline \multicolumn{6}{|c|}{ Sociodemográficas sobre engagement } \\
\hline Conductual & Estim. & Emocional & Estim. & Cognitivo & Estim. \\
\hline MUJER & $0,223 * * *$ & MUJER & $0,200 * * *$ & MUJER & $0,262 * * *$ \\
\hline HOMBRE & Ref. & HOMBRE & Ref. & HOMBRE & Ref. \\
\hline AUTÓCT & 0,036 & AUTÓCT. & $-0,205^{* * *}$ & AUTÓCT. & $-0,071 * *$ \\
\hline O.IMMIGRANTE & Ref. & O.IMMIGRANTE & Ref. & O.IMMIGRANTE & Ref. \\
\hline E.OBLIG. & $-0,048$ & E.OBLIG. & 0,036 & E.OBLIG. & 0,018 \\
\hline E.POS. & 0,057 & E.POS. & 0,045 & E.POS. & 0,008 \\
\hline E.UNIVER. & Ref. & E.UNIVER. & Ref. & E.UNIVER. & Ref. \\
\hline \multicolumn{6}{|c|}{ Sociodemográficas sobre ATL } \\
\hline Deportivas & Estim. & Rel_pantalla & Estim. & Culturales & Estim. \\
\hline MUJER & $-0,378 * * *$ & MUJER & 0,014 & MUJER & $0,200 * * *$ \\
\hline AUTÓCT. & $0,066 * *$ & AUTÓCT. & $0,188 * * *$ & AUTÓCT. & $-0,096 * *$ \\
\hline E.OBLIG. & $-0,097 * * *$ & E.OBLIG. & 0,008 & E.OBLIG. & $-0,093^{*}$ \\
\hline E.POS. & $-0,012$ & E.POS. & 0,025 & E.POS. & 0,055 \\
\hline E.UNIVER. & Ref. & E.UNIVER. & Ref. & E.UNIVER. & Ref. \\
\hline
\end{tabular}

Eng y ATL sobre PCB

Sociodemográficas sobre PCB

\begin{tabular}{cccc}
\hline & Estim. & & Estim. \\
\hline CONDUCT. & $\mathbf{0 , 2 4 1} \mathbf{1}^{* * *}$ & MUJER & $\mathbf{- 0 , 0 8 6 * * *}$ \\
\hline EMCIONAL & $\mathbf{- 0 , 1 5 4 * *}$ & AUTÓCT. & $\mathbf{0 , 3 1 0 * * *}$ \\
\hline COGNITIVO & 0,022 & E.OBLIG. & $\mathbf{- 0 , 2 2 5 * * *}$ \\
\hline REL_PAN & $\mathbf{0 , 1 1 9 *}$ & E.POS. & $\mathbf{- 0 , 1 1 3 * * *}$ \\
\hline CULTURAL & 0,023 & & \\
\hline DEPORTIVA & $-0,022$ & & \\
\hline$* p \leq 0,05 * * P \leq 0,01$ & $* * * P \leq 0,001$ & P-Value
\end{tabular}

Tabla 1. Efectos directos. Elaboración por los autores 
La Tabla 1 también indica las relaciones entre las variables sociodemográficas y los tres niveles de engagement. Aparecen diferencias significativas entre chicos y chicas: ellas tienen más engagement conductual, emocional y cognitivo que los chicos. El alumnado autóctono presenta un engagement emocional y cognitivo inferior al del alumnado de origen inmigrante. Un dato que destaca de esta parte de la tabla es que el nivel formativo familiar no tiene una relación significativa con ningún tipo de engagement. Es decir, parece que los estudios de los padres no afectan al nivel de esfuerzo y de constancia de sus hijos e hijas en la escuela.

Si nos fijamos en la relación entre las variables sociodemográficas y el tipo de actividades de tiempo libre, se ve cómo los chicos practican más actividades deportivas que las chicas; el alumnado autóctono practica más deporte que el de origen inmigrante y el alumnado con progenitores con estudios universitarios practica más deporte que el alumnado con padres con estudios obligatorios. Ni el sexo ni el nivel formativo familiar tienen un efecto sobre las actividades de relación y de pantalla, pero sí que aparece una diferencia significativa entre el alumnado autóctono y el de origen inmigrante, ya que los primeros practican más actividades de relación y de pantalla que los segundos. Por otro lado, las chicas practican más actividades culturales que los chicos, el alumnado autóctono menos que el alumnado inmigrante y el alumnado con padres con estudios obligatorios menos que el alumnado con padres con estudios universitarios.

Los resultados obtenidos corroboran de manera parcial la primera hipótesis, ya que solo las actividades de relación y de pantalla presentan un efecto directo significativo sobre el rendimiento académico, y es un efecto positivo. El modelo de ecuaciones estructurales ha permitido interpretar la desviación estándar que aparece en el rendimiento académico cuando se introduce la variable independiente de actividades de tiempo libre. Un cambio de desviación estándar en el predictor (ATL), produce un cambio en las unidades de desviación estándar de esta cantidad (el coeficiente) en el resultado (PCB). Siguiendo esta lógica, por cada unidad de desviación estándar que cambia el indicador de actividades de relación y de pantalla, la desviación del PCB aumenta un 0,119 . No obstante, este resultado hay que contextualizarlo. Según la literatura (Putnam, 2016) hay ciertas actividades de tiempo libre que establecen una relación funcional cuadrática con el rendimiento. Es decir, son actividades positivas si se practican hasta una cierta cantidad de horas. Si el alumnado se pasa de esta cantidad de horas, aparece un punto de inflexión y el efecto sobre el rendimiento pasa a ser negativo. Tal como se muestra en el Gráfico 2 en el Anexo, esta relación funcional cuadrática es el tipo de relación que aparece entre las actividades de relación y de pantalla y el rendimiento académico. Antes de controlar la relación por todas las variables del modelo, existía una relación funcional cuadrática pero, una vez entra en el modelo de ecuaciones estructurales, la relación pierde el carácter cuadrático y se convierte en lineal.

La segunda hipótesis también queda parcialmente confirmada. Las actividades de relación y de pantalla disminuyen el engagement conductual mientras que las actividades culturales lo aumentan. Por cada unidad de desviación de las actividades culturales -leer un libro por placer y/o la de tocar un instrumento musical- el engagement conductual aumenta un 0,134. En el caso del engagement emocional, las actividades de relación y de pantalla no tienen un efecto significativo sobre este tipo de engagement, pero tanto las actividades deportivas como las actividades culturales sí que presentan un efecto positivo significativamente. En relación al engagement cognitivo, 
los tres tipos de actividades tienen una relación significativa; mientras que las deportivas y culturales tienen un efecto positivo sobre este engagement, las actividades de relación y de pantalla muestran una relación negativa, ya que cada unidad de desviación de la participación en este tipo de actividades disminuye un 0,141 el nivel de esfuerzo en la escuela. Un resultado que encaja con la literatura sobre los beneficios del deporte y con la que señala los perjuicios de un uso abusivo de las nuevas tecnologías por parte de los y las jóvenes.

La Tabla 1 también muestra las relaciones entre el engagement, las actividades de tiempo libre y el rendimiento académico. En primer lugar, destaca que el engagement emocional esté asociado a un peor rendimiento académico, mientras que el engagement conductual se asocie a un mejor rendimiento. Por cada unidad de desviación del engagement conductual, el PCB incrementa un 0,241. Otro resultado sorpresivo de esta tabla es la falta de significatividad entre el rendimiento académico y el engagement cognitivo. En relación a las actividades de tiempo libre, tal como se ha indicado anteriormente, solo las actividades de relación y de pantalla muestran una relación significativa con el rendimiento académico.

\begin{tabular}{lc}
\hline \multicolumn{2}{c}{ EFECTOS INDIRECTOS } \\
\hline \multicolumn{1}{c}{ Sexo, engagement y PCB } \\
\hline PCB & $0,054^{* *}$ \\
\hline CONDUCTUAL & \\
\hline MUJER & $\mathbf{- 0 , 0 3 1 ^ { * }}$ \\
\hline PCB & \\
\hline EMOCIONAL & \\
\hline MUJER & $\mathbf{0 , 0 3 2 *}$ \\
\hline \multicolumn{1}{c}{ Origen inmigrante, engagement, ATL y PCB } \\
\hline PCB & \\
\hline EMOCIONAL & \\
\hline AUTÓCT & $\mathbf{0 , 0 2 2 *}$ \\
\hline PCB & \\
\hline REL_PANTALLA & \\
\hline AUTÓCTONO & $\mathbf{- 0 , 0 1 6 * *}$ \\
\hline PCB &
\end{tabular}

Tabla 2. Efectos indirectos entre variables. Elaboración por los autores

La Tabla 2 refuta la cuarta hipótesis porque no muestra una relación significativa que demuestre como las actividades de tiempo libre puedan incrementar el engagement y, de forma indirecta, aumentar el rendimiento académico del alumnado. La hipótesis cinco también queda anulada porque no se puede comprobar que la incidencia de las 
actividades de tiempo libre sobre el engagement y sobre el rendimiento académico sea mayor cuando la relación entre el origen social y el rendimiento es más negativa.

Esta tabla aporta otros resultados interesantes. La primera relación indirecta indica que el hecho de tener un engagement de conducta alto es más positivo para las chicas que para los chicos. Por cada unidad de desviación del engagement conductual, el PCB de las chicas aumenta un 0,054 en relación a los chicos. En este sentido, la tercera hipótesis, la que apuntaba que el engagement puede compensar el efecto de las variables sociodemográficas sobre el rendimiento académico, es cierta. Las chicas tienen peores resultados que los chicos a no ser que presenten el mismo nivel de engagement de conducta que ellos. Cuando tanto chicos como chicas tienen un engagement conductual alto, las chicas sacan mejores notas. El engagement emocional presenta una relación indirecta inversa: tener un alto engagement emocional es más beneficioso para los chicos que para las chicas en relación a la media de notas. En este caso, por cada unidad de desviación del engagement emocional, el rendimiento académico de los chicos sube un 0,031 respecto al de las chicas. Es decir, los chicos con un engagement emocional alto tienen un rendimiento académico superior al de las chicas con el mismo nivel de engagement emocional.

Una relación indirecta significativa se encuentra entre la PCB, el engagement emocional y el origen inmigrante. La Tabla 2 indica que el alumnado autóctono con engagement emocional tiene una media de notas superior a la del alumnado de origen inmigrante con el mismo nivel de engagement emocional. Por cada unidad de desviación estándar del engagement emocional, la PCB del alumnado autóctono aumenta un 0,032 respecto a la del alumnado de origen inmigrante. Otra relación indirecta relevante es la que se establece entre la PCB, las actividades de tiempo libre y la variable de origen inmigrante. La Tabla 2 indica que el alumnado autóctono con una alta participación en actividades de relación y de pantalla tiene un rendimiento académico superior al que tiene el alumnado de origen inmigrante con el mismo nivel de participación en este tipo de actividades.

Al final de la Tabla 2, aparece otro efecto compensador del engagement conductual. Se observa cómo el alumnado de origen inmigrante saca mejores notas que el alumnado autóctono, cuando ambos practican actividades de relación y de pantalla y tienen un engagement conductual positivo. Por cada unidad de desviación del engagement conductual y de las actividades de relación y de pantalla, la PCB del alumnado de origen inmigrante aumenta un 0,016 respecto a la del alumnado autóctono. Un dato que vuelve a confirmar la tercera hipótesis, la del potencial compensador del engagement escolar sobre el efecto del origen social en el rendimiento académico de las personas jóvenes.

\section{CONCLUSIONES}

Esta investigación ha aportado información valiosa para el estudio de las actividades de tiempo libre, del engagement escolar y del rendimiento académico de las personas jóvenes. Entre los resultados esperados, se ha confirmado que el alumnado con padres con estudios universitarios tiene más engagement conductual que el alumnado con padres con estudios obligatorios; que las chicas presentan un engagement conductual, emocional y cognitivo más alto que el de los chicos, y que el alumnado de origen 
inmigrante tiene un engagement emocional y cognitivo superior al del alumnado autóctono. También se ha corroborado la idea de que el engagement conductual tiene un efecto positivo sobre el rendimiento académico del alumnado. Sin embargo, se ha visto cómo el engagement emocional puede tener un efecto negativo sobre el rendimiento, una conclusión sorprendente que invita a seguir ahondando en la relación entre estas dos variables.

A lo largo del estudio han aparecido otros resultados sorpresivos que sería interesante seguir investigando. En primer lugar, se ha observado cómo las chicas de $4^{\circ}$ de ESO de Barcelona tienen un rendimiento académico ligeramente inferior al de los chicos (si se mide a través de la PCB), a no ser que tengan el mismo nivel de engagement conductual. La media de notas de las chicas solo es superior a la de los chicos cuando asisten a clase de manera regular y se comportan con disciplina dentro del aula. En segundo lugar, según los resultados obtenidos, parece que los estudios de los progenitores no afectan al nivel de esfuerzo y de constancia de sus hijos e hijas en la escuela. El tercer resultado sorprendente es que las actividades culturales no muestran un efecto directo sobre el rendimiento académico. Sin embargo, sí que afectan positivamente a los tres tipos de engagement, incluido el de conducta, que está asociado a un mejor rendimiento académico. Esta diferencia de efectos que tienen las actividades culturales muestra cómo el engagement escolar y el rendimiento académico siguen lógicas diferentes. Otro ejemplo de esta diferencia nos la dan las actividades de relación y de pantalla. Como se ha observado, son las únicas actividades de tiempo libre que tienen un efecto positivo sobre el rendimiento del alumnado, pero presentan un efecto negativo sobre su engagement conductual y cognitivo.

En relación a las actividades deportivas, se ha confirmado que tienen un efecto positivo sobre el engagement emocional y el cognitivo. Por consiguiente, los chicos y chicas que hacen ejercicio para mantenerse en forma o practican un deporte tenderán a esforzarse más en clase, a dar lo mejor de sí, a entregar los deberes a tiempo y a valorar positivamente su experiencia en la escuela. No obstante, las actividades deportivas no presentan un efecto significativo en relación al engagement de conducta, ni en relación al rendimiento académico. Unos resultados que aportan más matices al debate sobre los beneficios del deporte y, al mismo tiempo, invitan a seguir estudiando sus efectos entre la población joven.

Las relaciones indirectas han señalado dos efectos de compensación del engagement conductual sobre el peso del origen social en el logro escolar del alumnado. Por un lado, el engagement conductual compensa la desigualdad educativa causada por el sexo, en el sentido que tiene un efecto más positivo entre las chicas que entre los chicos. Por otro lado, se ha visto como el engagement conductual también compensa el peso del origen inmigrante. Aunque los autóctonos tienen una media de notas superior a la del alumnado de origen inmigrante, cuando los dos grupos tienen el mismo nivel de engagement conductual y practican actividades de rerlación y de pantalla, son los y las jóvenes de origen inmigrante quienes sacan mejores notas.

Es importante reconocer las limitaciones empíricas de este trabajo. El modelo de ecuaciones estructurales ha sido una técnica adecuada para medir el efecto de las actividades de tiempo libre sobre el rendimiento académico y sobre el engagement escolar. No obstante, la dirección de tales efectos se ha orientado basándose en la teoría. Es decir, no se ha podido establecer una asociación de causa-efecto cien por cien segura. Esta cortapisa de la investigación podría superarse a través de un análisis 
longitudinal. Otra limitación del presente trabajo está asociada a las actividades deportivas. Las preguntas del cuestionario que aludían a estas actividades estaban orientadas a saber si los y las jóvenes hacían ejercicio para mantenerse en forma o si practicaban algún deporte. Solo se les preguntaba por la frecuencia, pero no por el tipo de deporte que practicaban. Seguramente, con una información más concreta sobre las prácticas deportivas de la población joven, aparecerían más efectos significativos entre las variables del modelo.

Si bien las actividades de tiempo libre no necesitan una justificación instrumental, ya que por naturaleza son actividades orientadas al descanso, al entretenimiento o a la formación desinteresada, es importante destacar sus efectos en el ámbito formativo, con el fin de tener la posibilidad de orientar medidas políticas que promuevan aquellas actividades con un impacto positivo en las experiencias escolares de la población joven y, al mismo tiempo, potencien aquellas actividades con capacidad de compensación del peso del origen social sobre su rendimiento académico. De la misma manera, podrían plantearse políticas de juventud orientadas a mejorar el engagement conductual entre el alumnado, porque es un tipo de engagement que ha destacado como mecanismo atenuador del efecto de las variables sociodemográficas sobre el logro escolar, y también porque mejora las experiencias escolares y el rendimiento académico de todo el alumnado. Una manera de incrementar el engagement conductual, tal como ha demostrado esta investigación, sería motivando a las personas jóvenes a practicar actividades culturales, como leer por placer o tocar un instrumento fuera del horario escolar.

\section{BIBLIOGRAFIA}

Appleton, J.J. y otros (2006). Measuring cognitive and pgsycological engagement: validation of the student engagement instrument. Journal of School Psychology, 44 (5), 427-445.

Benedicto, J. y otros (2017). Informe Juventud España 2016. Madrid: Instituto de la Juventud.

Boekaerts, M. (2016). Engagement as an inherent aspect of the learning process. Learning and Instruction, 43, 76-83. doi: 10.1016/j.learninstruc.2016.02.001

Byrne, B.M. (2001). Structural equation modeling with AMOS: Basic concepts, applications, and programming. NJ: Erlbaum.

Dumazedier, J. (1968). Hacia una civilización del ocio. Barcelona: Estela.

Dumazedier, J. y otros (1971). Ocio y sociedad de clases. Barcelona: Fontanella.

Eccles, J. y Gootman, J. (2002). Community programs to promote youth development. Washington DC: The National Academies Press.

Eccles, J., Barber, B., Stone, M. y Hunt, J. (2003). Extracurricular Activities and Adolescent Development. Journal of Social Issues, 59, 865-89.

Finn, J.D. (1989). Withdrawing from School. Review of Educational Research Summer, 59 (2),117-142.

Finn, J. y Voelkl, K. ( 1993). School Characteristics Related to Student Engagement. The Journal of Negro Education, 62 (3), 249-268. https://doi.org/10.2307/2295464

Finn, J. y Rock, D. (1997). Academic success among students at risk for school failure. Journal of Applied Psychology, 82 (2), 221-234. doi: 10.1037/0021-9010.82.2.221

Finn, J. y Zimmer, K. (2012). Student engagement: What is it? Why does it matter? En S.L. Christenson, A.L. Reschly y C. Wylie (coords.), Handbook of research on student engagement (pp. 97-131). New York: Springer Science Business Media. doi: 10.1007/9781-4614-2018-7_5 
Fraguela, R., De-Juanas, A. y Franco, R. (2017). Ocio deportivo en jóvenes potencialmente vulnerables: beneficios percibidos y organización de la práctica. Pedagogía Social, 31, 4958.

Fredricks, J. y Eccles, J. (2006). Is Extracurricular Participation Associated with Beneficial Outcomes? Concurrent and Longitudinal Relations. Developmental Psychology, 42, 698713.

Fredricks, J., Blumenfeld, P.C. y Paris, A. (2004). School Engagement: Potential of the Concept, State of the Evidence. Review of Educational Research, 74 (1), 59109. https://doi.org/10.3102/00346543074001059

Janosz, M., Archambault, I., Morizot, J. y Pagani, L.S. (2008). School Engagement Trajectories and Their Differential Predictive Relations to Dropout. Journal of Social Issues, 64 (1), 2140. doi: https://doi.org/10.1111/j.1540-4560.2008.00546.x

Jimerson, S., Campos, E. y Greif, J. (2003). Toward an Understanding of Definitions and Measures of School Engagement and Related Terms. The California School Psychologyst, 8, 7-29.

Juvonen, J., Espinoza,G. y Knifsend, C. ( 2012). The Role of Peer Relationships in Student Academic and Extracurricular Engagement. Handbook of Research on Student Engagement, 387-403

Kline, R.B. (1998). Principals and practices of structural equation modeling. New York, NY: Guilford Press.

López-Roldán, P. y Fachelli, S. (2015). Clasificación de las técnicas de análisis de datos. En P. López-Roldán y S. Fachelli, Metodología de la Investigación Social Cuantitativa. Bellaterra: Dipòsit Digital de Documents, Universitat Autònoma de Barcelona.

Merino, R. (2007). Pathways from school to work: can the competences acquired in leisure activities improve the construction of pathways. Journal of Education and Work, 20 (2), 139-159.

Moreno Colom, S. (2009). Uso del tiempo, desigualdades sociales y ciclo de vida. Política y Sociedad. 46 (3), 191-202.

Nordlander, E. (2016). Are Young People's Activites an Important Link between Class Origin and Educational Attainment? European Sociological Review, 32 (2), 238-250.

Putnam, R. (2015). Our Kids. The American Dream in Crisis. New York: Simon \& Schuster.

Ramos-Díaz, E., Rodríguez-Fernández, A., Ros, I. y Antonio-Aguirre, I. (2016). Implicación escolar y autoconcepto multidimensional en una muestra de estudiantes españoles de secundaria. Revista complutense de educación, $28 \quad$ (4), 1.1031.118. http://dx.doi.org/10.5209/RCED.51600

Reeve, J. y Tseng, C. (2011). Agency as a fourth aspect of students’ engagement during learning activities. Contemporary Educational Psychology, 36 (4), 257-267. doi: https://doi.org/10.1016/j.cedpsych.2011.05.002

Shildrick, T.A. y MacDonald, R. (2006): In defence of subculture: young people, leisure and social divisions. Journal of Youth Studies, 9 (2),125-140.

Tarabini, A., Curran, M., Montes, A. y Parcerisa, Ll. (2015). La vinculación escolar como antídoto del abandono escolar prematuro: explorando el papel del habitus institucional. Profesorado: revista de currículum y formación del profesorado, 19 (3), 196-212.

Veblen, T. (2004). Teoria de la clase ociosa. Madrid: Alianza Editorial.

Viñals Blanco, A. (2016). El ocio conectado, móvil, transmedia y multisoporte de los jóvenes en la era digital. Fonseca, Journal of Communication, 13, 99-113.

Voelkl, K.E. (1997). Identification with school. American Journal of Education, 105 (3), 294-318

Zaff, J., Moore, K., Pappillo, A. y Williams, S. (2003). Implications of extracurricular activity participation during adolescence on positive outcomes. Journal of Adolescent Research, 18, 599-630. 


\section{ANEXO}

\begin{tabular}{|c|c|c|c|c|}
\hline & & & $\mathrm{N}$ & $\%$ \\
\hline \multirow{3}{*}{ Sexo } & & & 2.056 & 100 \\
\hline & Hombre & & 1.087 & 52,9 \\
\hline & Mujer & & 969 & 47,1 \\
\hline \multirow[t]{4}{*}{ Nivel Formativo Familiar } & & & 1.999 & 100 \\
\hline & Estudios obligatorios & & 667 & 33,4 \\
\hline & Estudios postobligatorios & & 516 & 25,8 \\
\hline & Estudios universitarios & & 816 & 40,8 \\
\hline \multirow[t]{4}{*}{ Origen inmigrante } & & & 1.916 & 100 \\
\hline & Autóctono & & 1.470 & 76,7 \\
\hline & Origen inmigrante & & 446 & 23,3 \\
\hline & Mínimo & Máximo & $\mathbf{N}$ & Media \\
\hline Actividades de relación y de pantalla & 0 & 10 & 2.053 & 5,794845 \\
\hline Actividades deportivas & 0 & 10 & 2.053 & 5,053575 \\
\hline Actividades culturales & 0 & 10 & 2.053 & 2,727244 \\
\hline Engagement de conducta & 0 & 10 & 1.996 & 6,1117 \\
\hline Engagement emocional & 0 & 10 & 1.996 & 5,6853 \\
\hline Engagement cognitivo & 0 & 10 & 1.996 & 5,8652 \\
\hline
\end{tabular}

Tabla 3. Estadísticos descriptivos de las variables utilizadas

\section{Centros públicos}

Centros concertados

$\%$ alumnos

alumnos \% alumnos centros alumnos \% alumnos

\begin{tabular}{lccccccccc}
\hline ALTA COMPLEJIDAD & 4 & 316 & 15,37 & 4 & 163 & 7,93 & 8 & 479 & 23,30 \\
\hline COMPLEJIDAD MEDIA & 6 & 517 & 25,15 & 8 & 782 & 38,04 & 14 & 1299 & 63,18 \\
\hline BAJA COMPLEJIDAD & 2 & 93 & 4,52 & 3 & 185 & 9,00 & 5 & 278 & 13,52 \\
\hline TOTAL & $\mathbf{1 2}$ & $\mathbf{9 2 6}$ & $\mathbf{4 5 , 0 4}$ & $\mathbf{1 5}$ & $\mathbf{1 1 3 0}$ & $\mathbf{5 4 , 9 6}$ & $\mathbf{2 7}$ & $\mathbf{2 0 5 6}$ & $\mathbf{1 0 0}$ \\
\hline
\end{tabular}

Tabla 4. Número total de centros y de alumnos que participaron en el proyecto ISCY 


\begin{tabular}{lccc}
\hline & Pantalla & Deporte & Cultura \\
\hline Navegar por internet & 0,755 & & \\
\hline Mirar la TV & 0,639 & & \\
\hline $\begin{array}{l}\text { Pasar el rato con los } \\
\text { amigos }\end{array}$ & 0,629 & 0,922 & \\
\hline $\begin{array}{l}\text { Practicar un deporte } \\
\text { Hacer ejercicio para } \\
\text { mantenerse en forma }\end{array}$ & 0,913 & 0,770 \\
\hline $\begin{array}{l}\text { Tocar un instrumento } \\
\text { musical }\end{array}$ & & & 0,742 \\
\hline \begin{tabular}{l} 
Leer un libro por placer \\
\hline
\end{tabular}
\end{tabular}

Tabla 5. Matriz de componentes rotados del Análisis factorial

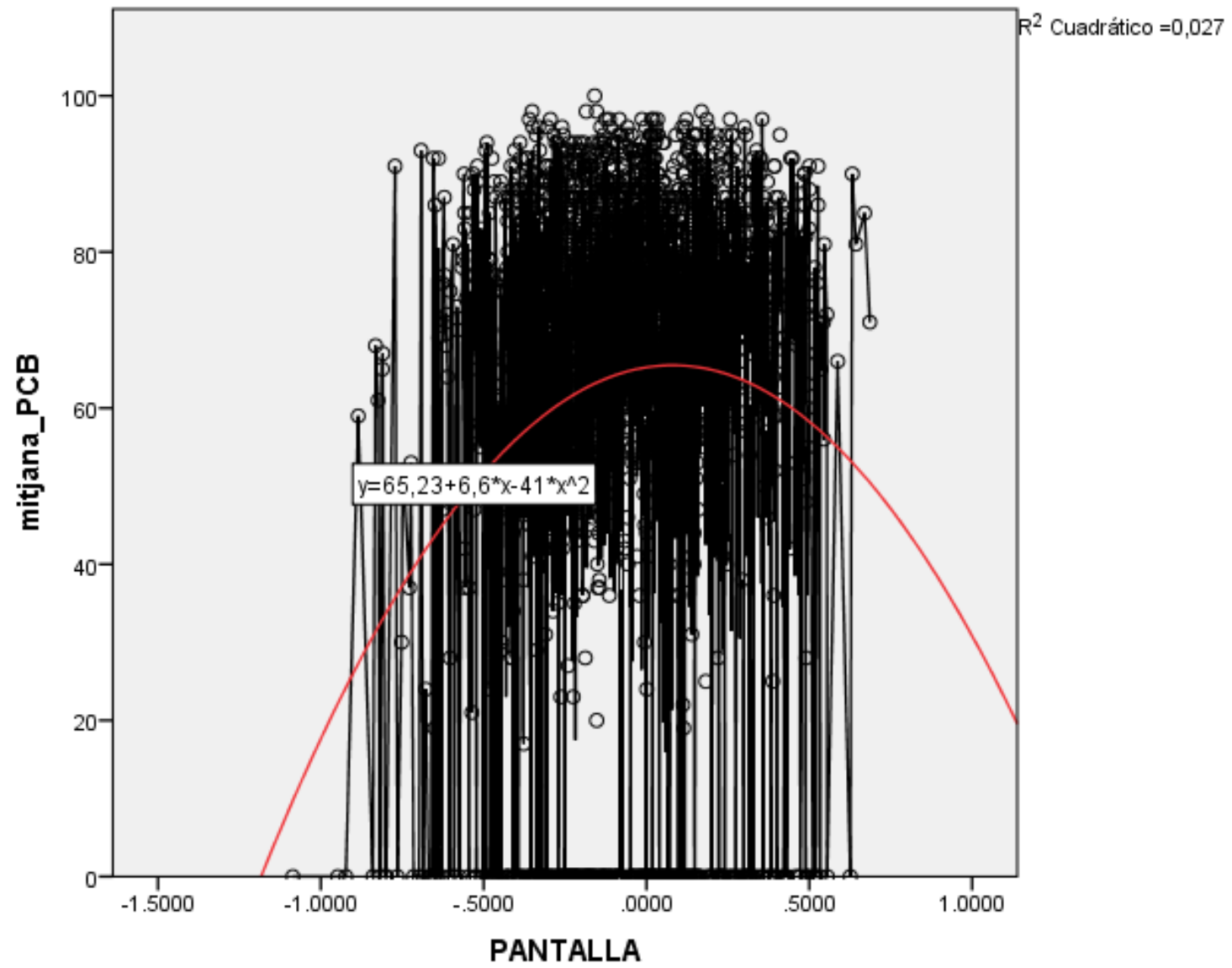

Figura 2. Relación cuadrática entre PCB y Actividades de relación y de pantalla 


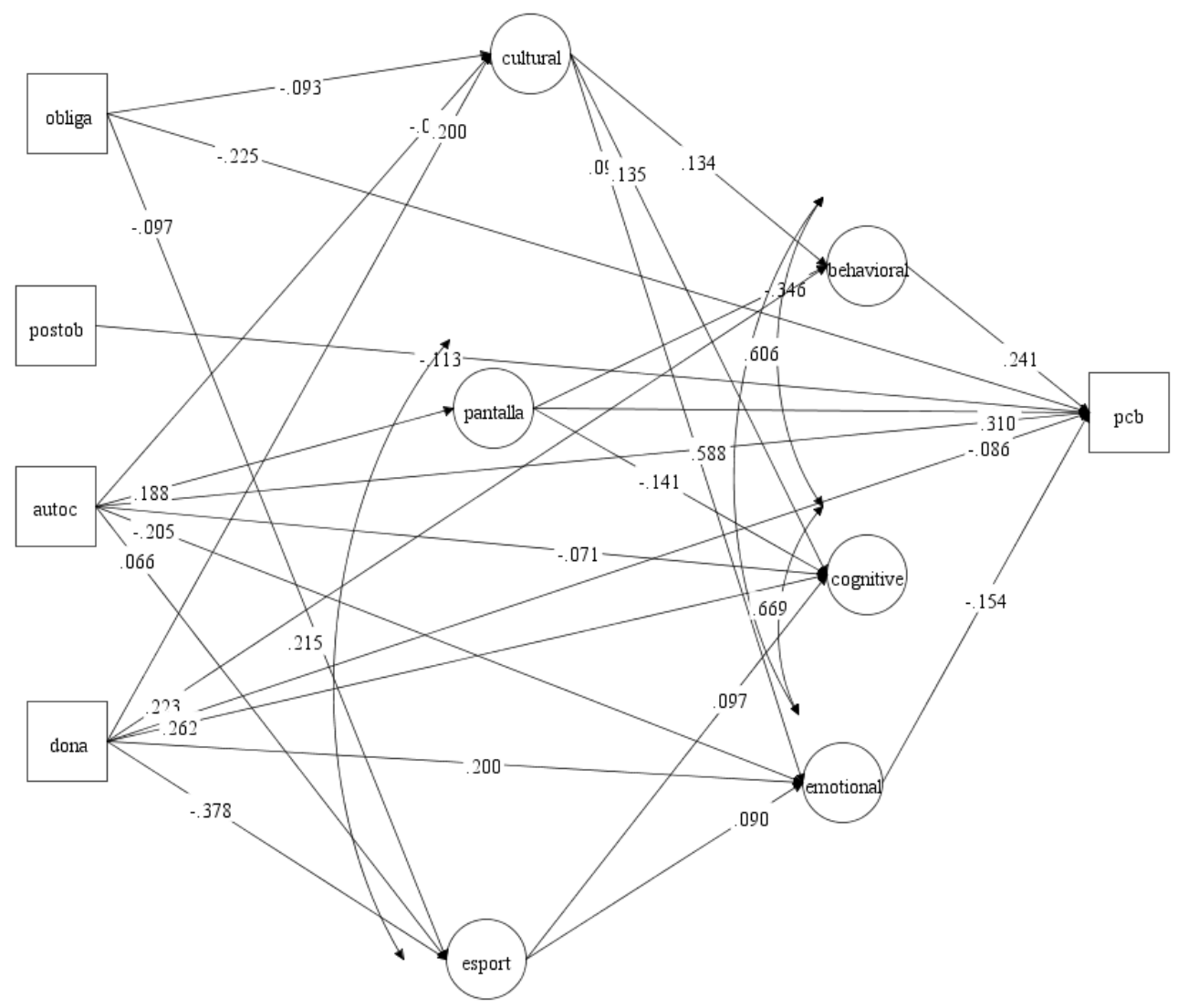

Figura 3. Diagrama de los efectos directos e indirectos entre variables 